\title{
Ventricular Septal Rupture After Recent Myocardial Infarction in the Very Elderly Clinical Implication for Surgery
}

\author{
Riku Arai, ${ }^{1}$ MD, Daisuke Fukamachi, ${ }^{1}$ MD, Naotaka Akutsu, ${ }^{1}$ MD, \\ Yasuo Okumura, ${ }^{1}$ MD and Masashi Tanaka, ${ }^{2}$ MD
}

\begin{abstract}
Summary
Ventricular septal rupture (VSR) is one of the fatal complications of myocardial infarction in the percutaneous coronary intervention era. A rapid diagnosis, medical and mechanical support, and surgical intervention are required for recovery and survival. In such a situation, the risk of complications associated with surgery is very high, especially in very elderly patients, in which any therapeutic strategy should be carefully discussed by the heart team. Herein, we describe two cases of VSRs after recent myocardial infarction (RMI) in very elderly patients that required debate regarding whether to perform surgery. The patients included a 93-year-old man and 89-year-old man, both of which were not highly frail before the RMI occurred. In the former case, a conservative strategy was adopted because the risk of surgery was considered, but he did not survive. On the other hand, the latter patient underwent surgery and his life was ultimately saved. Based on these two cases, we concluded that even if the patients are very old, if possible, surgical intervention should be fully considered.
\end{abstract}

Key words: Mechanical complication, Therapeutic strategy, Frail

(Int Heart J 2020; 61: 831-837)

$\mathrm{V}$ entricular septal rupture (VSR) is a fatal complication of myocardial infarction (MI). Though rapid diagnosis, medical and mechanical support, and surgical intervention are needed to recover and survive from VSR, a conservative strategy might be taken into consideration due to the high surgical risk, especially in the very elderly. We herein experienced two cases of VSR after MI in very elderly patients, of which one received a conservative strategy and the other underwent surgery.

\section{Case Reports}

A 93-year-old man, who was not highly frail, with type 2 diabetes presented to the emergency department of our hospital complaining of fatigue and poor appetite from the previous day. On admission, he had chest discomfort, and his vital signs on admission were as follows: blood pressure $121 / 75 \mathrm{mmHg}$, heart rate 90 beats per minute, and $\mathrm{SpO}_{2} 98 \%\left(\mathrm{FiO}_{2}\right.$ 0.21). Cardiac and pulmonary auscultation were unremarkable. His lactate level was high $(7.4 \mathrm{mmol} / \mathrm{L})$. A 12-lead electrocardiogram (ECG) during the initial examination revealed sinus rhythm with an elevated ST-segment in leads V1 through V6 and Qwaves in V1 through V5 and II, III, and aVF (Figure 1). Transthoracic echocardiography (TTE) revealed a reduced left ventricular ejection fraction (LVEF), estimated to be about $35 \%-45 \%$, with anteroseptal and apical wall hypokinesis without D-shape, echo-free space, or valvular disease. A chest X-ray revealed no notable findings, including congestion. The laboratory data revealed increased creatinine kinase (CK; $1686 \mathrm{U} / \mathrm{L})$ and troponin I serum levels (more than $50 \mathrm{ng} / \mathrm{mL}$ ). A recent MI (RMI) was diagnosed, and an emergent coronary angiography (CAG) was performed due to lasting chest symptoms. It revealed no significant stenosis in the right coronary (Figure 2A) and left circumflex arteries, but a total occlusion in the mid left anterior descending artery (LAD; Figure 2B), which coincided with the culprit inferred from the 12-lead ECG and echocardiography. We immediately moved to a primary percutaneous coronary intervention of the mid LAD with a drug-eluting stent with a Thrombolysis in Myocardial Infarction (TIMI) grade 2 flow (Figure 2C). An intra-aortic balloon pump (IABP) was placed because hemodynamics became temporarily unstable during catheter examination. After that, the patient's chest symptoms improved and the CK levels peaked at $2741 \mathrm{U} / \mathrm{L}$. He remained asymptomatic and hemodynamically stable during the first two days of hospitalization, with the CK levels following a downward trend. On the third day, he developed fatigue accompanied by hemodynamic instability with lactic acidosis, elevated liver enzymes, and kidney dysfunction. Cardiac auscultation revealed a newly pansystolic murmur at the left lower sternal edge. A chest X-

From the ${ }^{1}$ Division of Cardiology, Department of Medicine, Nihon University School of Medicine, Tokyo, Japan and ${ }^{2}$ Department of Cardiovascular Surgery, Nihon University School of Medicine, Tokyo, Japan.

Address for correspondence: Yasuo Okumura, MD, Division of Cardiology, Department of Medicine, Nihon University School of Medicine, 30-1 Oyaguchijomachi, Itabashi-ku, Tokyo 173-8610, Japan. E-mail: okumura.yasuo@nihon-u.ac.jp

Received for publication January 3, 2020. Revised and accepted April 6, 2020.

doi: 10.1536/ihj.20-003

All rights reserved by the International Heart Journal Association. 


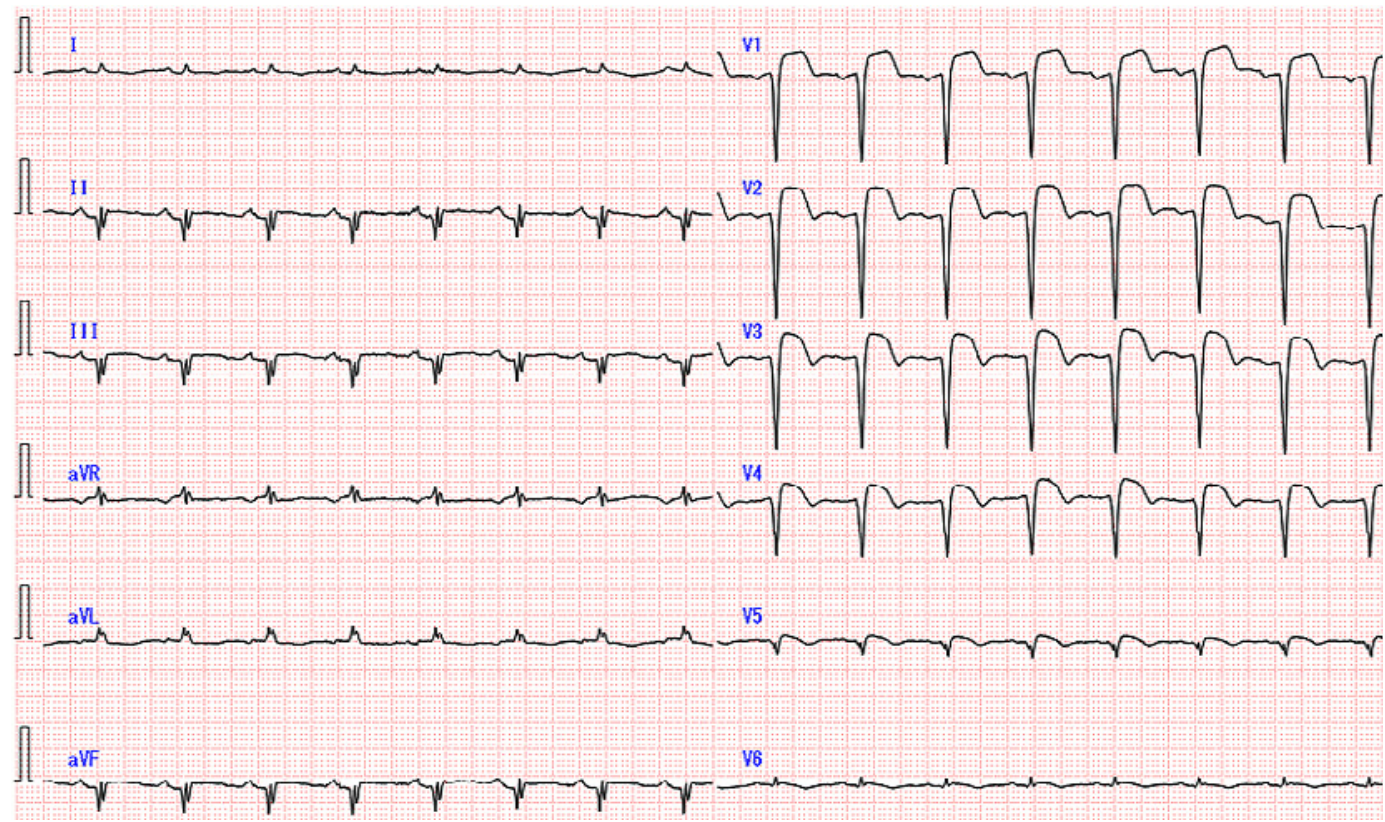

Figure 1. Electrocardiogram on admission showing sinus rhythm, left axis deviation, and QS complexes with ST-segment elevation in leads V1-V5.
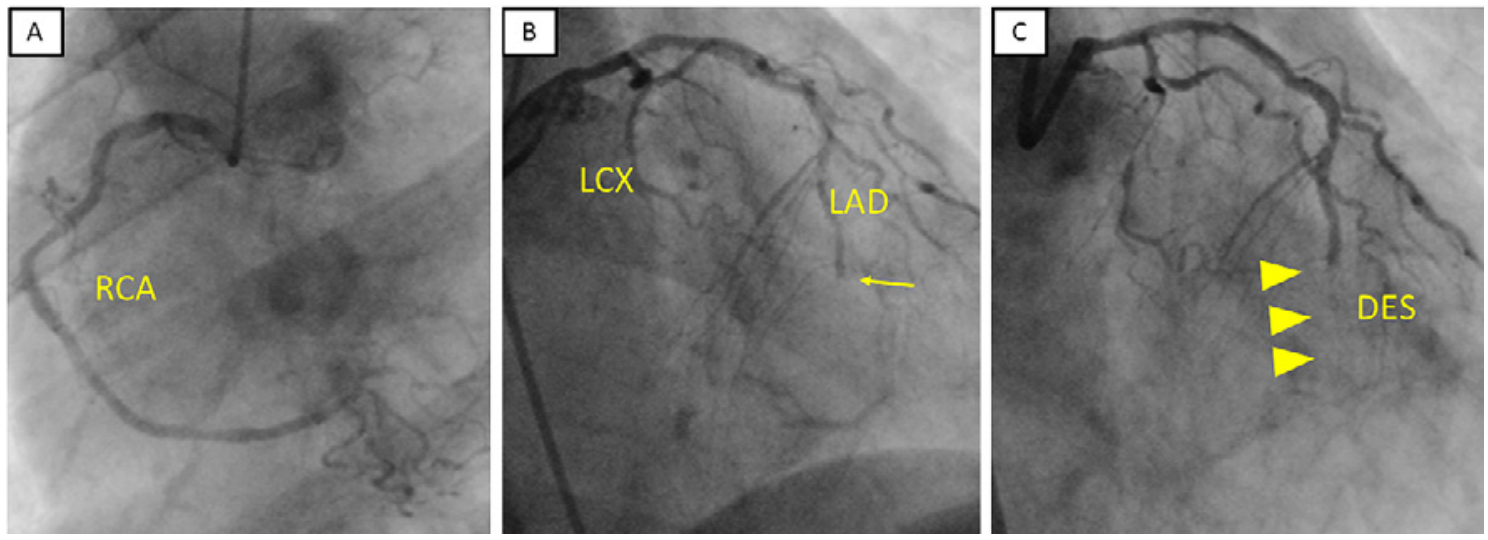

Figure 2. Coronary angiography. A: Left anterior oblique (LAO) view: right coronary artery (RCA) with no significant stenosis. B: Right anterior oblique (RAO) cranial view before percutaneous coronary intervention (PCI): left anterior descending artery (LAD) with a total occlusion in the mid portion (arrow) and left circumflex artery (LCX) with no significant stenosis. C: RAO cranial view after the PCI: a drug-eluting stent (DES) was placed in the mid LAD with a Thrombolysis in Myocardial Infarction (TIMI) grade 2 flow (arrow).

ray revealed congestion. A TTE revealed the presence of a ventricular septal defect on the apical side (Figure 3A), and color Doppler revealed a shunt between the left and right ventricles through the septal defect (Figure 3B). The pulmonary artery systolic pressure was elevated (65 $\mathrm{mmHg}$ ). We diagnosed the patient with cardiogenic shock due to the occurrence of a VSR after RMI. The patient was initiated on inotropic agents. After optimal medical and mechanical support including inotropic agents and IABP was performed, it was discussed with the heart team whether to perform surgical intervention. Because the patient's surgical complication risk was very high (EuroSCORE II: 23.4\%; JapanSCORE: 30-day operative mortality [CABG only/valve] $56.2 \% / 23.9 \%$; and 30-day operative mortality plus major complications [CABG only/ valve] $58.5 \% / 61.6 \%$ ) (Table), and he and his family were not willing to have him undergo surgery after our explanation of the risks and benefits of surgery, and a conservative strategy was finally selected. He recovered from the shock due to the optimal medical treatment, and we were able to remove the IABP on day 7. However, on day 9 , he went into cardiac arrest from a sudden bradycardia and died.

We subsequently experienced a similar case of an 89 year-old, who was not highly frail, with hypertension and xeroderma and a history of cellulitis, who presented to the emergency department of our hospital complaining of poor appetite from three days prior. On admission, he was in shock with perspiration, and his vital signs on admission were as follows: blood pressure $78 / 47 \mathrm{mmHg}$, heart 

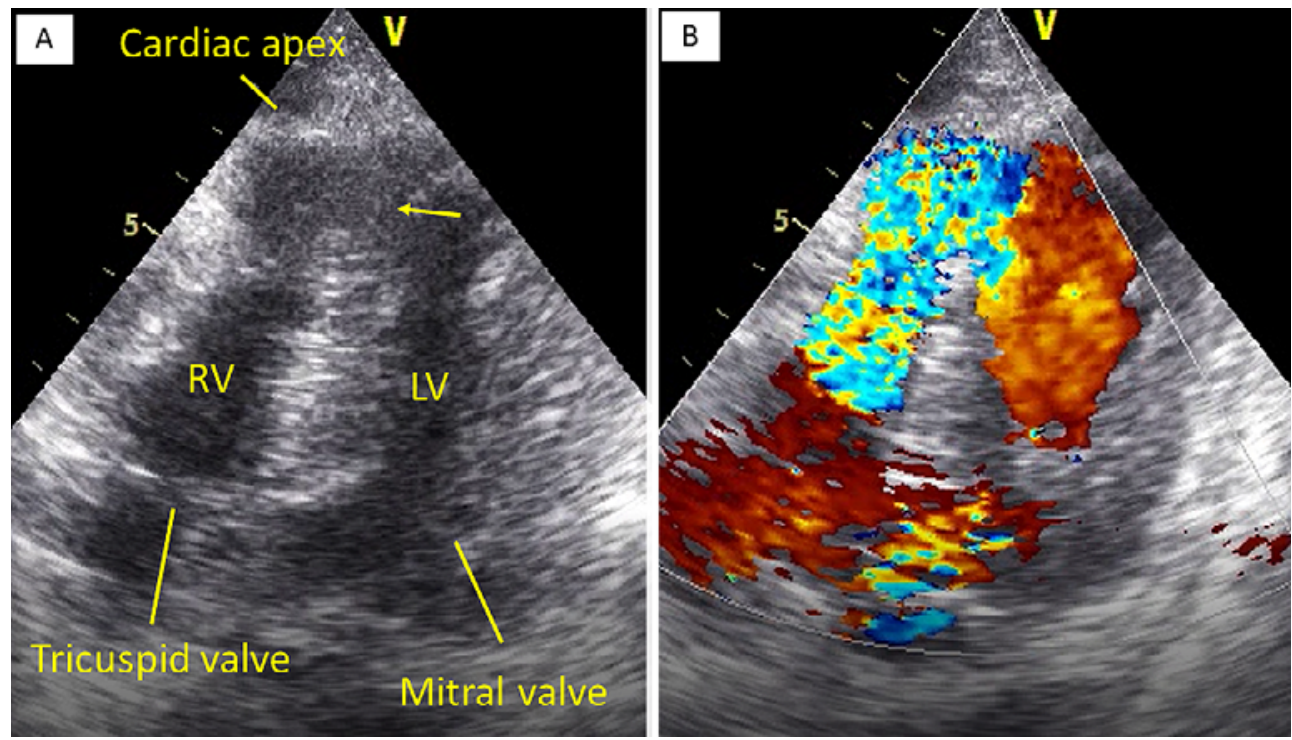

Figure 3. Transthoracic echocardiography. A: Presence of a ventricular septal defect as seen in the apical four-chamber view (arrow). B: Color Doppler showing turbulent flow (mosaic) from the left ventricle (LV) to right ventricle $(\mathrm{RV})$ through the interventricular septal defect.

Table. Patient Characteristics

\begin{tabular}{|c|c|c|}
\hline & Case 1 & Case 2 \\
\hline Age (years), sex & 93, male & 89 , male \\
\hline Height $(\mathrm{cm})$ & 160 & 163 \\
\hline Body weight $(\mathrm{kg})$ & 66.5 & 47.8 \\
\hline Body mass index $\left(\mathrm{kg} / \mathrm{m}^{2}\right)$ & 26 & 18 \\
\hline GNRI & 98.4 & 77.3 \\
\hline CSHA-CFS & 3 & 4 \\
\hline Cognitive function & Preserved & Preserved \\
\hline \multicolumn{3}{|l|}{ Vital Signs at the initial examination } \\
\hline Blood Pressure (mmHg) & $121 / 75$ & $78 / 47$ \\
\hline Heart rate $(\mathrm{bpm})$ & 90 & 93 \\
\hline Respiratory rate (breaths/minute) & 18 & 24 \\
\hline Body temperature $\left({ }^{\circ} \mathrm{C}\right)$ & 36.6 & 36.8 \\
\hline Consciousness level & clear & JCS II- 10 \\
\hline \multicolumn{3}{|l|}{ Laboratory data at the initial examination } \\
\hline Lactate $(\mathrm{mmol} / \mathrm{L})$ & 7.4 & 9.2 \\
\hline Albumin (mg/dL) & 3.3 & 2.9 \\
\hline Creatinine (mg/dL) & 1.25 & 1.4 \\
\hline Troponin I (ng/mL) & $\geq 50$ & $\geq 50$ \\
\hline CK/CK-MB (U/L) & $1686 / 113$ & $1001 / 34$ \\
\hline Max CK/CK-MB (U/L) & $2741 / 148$ & $1001 / 34$ \\
\hline \multicolumn{3}{|l|}{ Surgical complication risks } \\
\hline EuroSCORE II $(\%)$ & 23.4 & 21.3 \\
\hline \multicolumn{3}{|l|}{ JapanSCORE $(\%)$} \\
\hline 30-day operative mortality (CABG only/Valve) & $56.2 / 23.9$ & $13.4 / 68.0$ \\
\hline 30-day operative mortality plus major complications (CABG only/Valve)* & $58.5 / 61.6$ & $52.6 / 88.3$ \\
\hline \multicolumn{3}{|l|}{ Characteristics of a ventricular septal rupture } \\
\hline Timing of the diagnosis as a VSR from the onset of an AMI & 4 days & 7 days \\
\hline Qp/Qs & - & 1.5 \\
\hline Pulmonary artery systolic pressure $(\mathrm{mmHg})$ & 65 & 49 \\
\hline Morphological type of VSR & Simple or complex & Simple \\
\hline
\end{tabular}

* The risk included 30-day operative mortality as well as major complications. GNRI indicates geriatric nutritional risk index; CSHA-CFS, Canadian Study of Health and Aging Clinical Frailty Scale; JCS, Japan Coma Scale; CK, creatine kinase; CK-MB, creatine kinase mascular brain; CABG, coronary artery bypass graft; VSR, ventricular septal rupture; and AMI, acute myocardial infarction. 


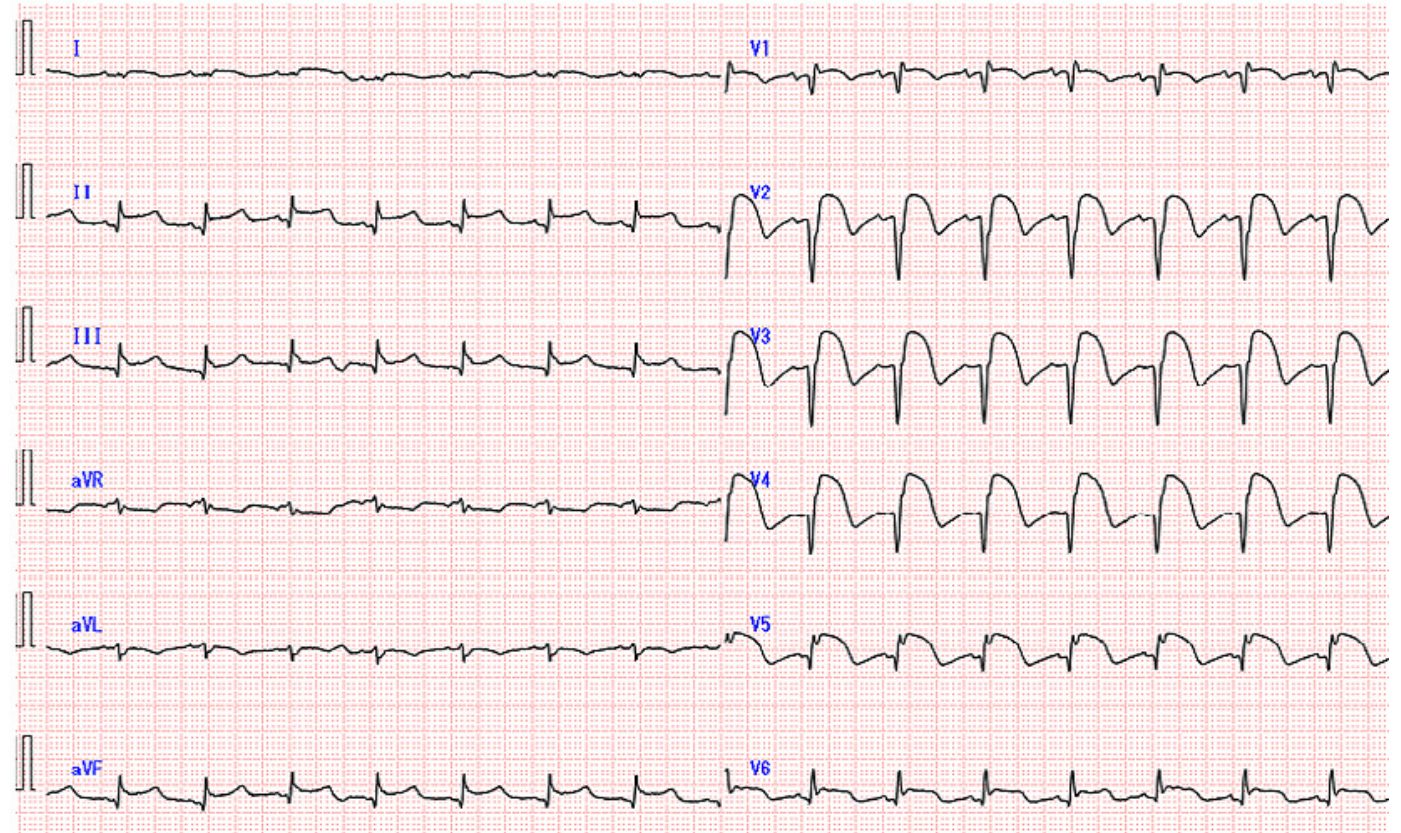

Figure 4. Electrocardiogram on admission showing sinus rhythm and abnormal Q-waves with ST-segment elevation in leads V1-V5 and II, III, and aVF.
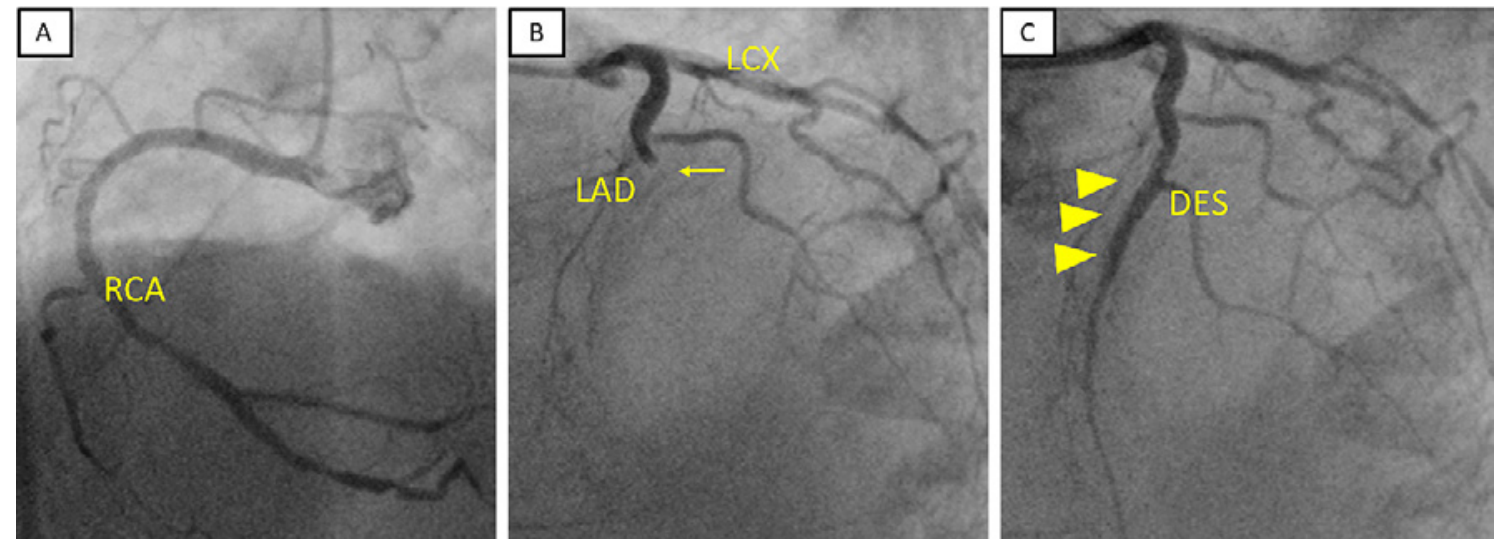

Figure 5. Coronary angiography. A: LAO view: right coronary artery (RCA) with no significant stenosis. B: LAO cranial view before percutaneous coronary intervention (PCI): left anterior descending artery (LAD) with a total occlusion in the mid portion (arrow) and left circumflex artery (LCX) with no significant stenosis. C: LAO cranial view after PCI: a drug-eluting stent (DES) was placed in the mid LAD with a thrombolysis in myocardial infarction (TIMI) grade 3 flow (arrow).

rate 93 beats per minute, and $\mathrm{SpO}_{2} 98 \%\left(\mathrm{FiO}_{2}\right.$ 1.0). Cardiac and pulmonary auscultation were unremarkable. His lactate level was high $(9.2 \mathrm{mmol} / \mathrm{L})$. The 12-lead ECG during the initial examination revealed sinus rhythm, with an elevated ST-segment in leads V1 through V6, and II, III, and aVF and Q-waves in V1 through V5 (Figure 4). TTE revealed a reduced LVEF, estimated to be about $30 \%-40 \%$, with anteroseptal and apical wall hypokinesis without a D-shape, echo-free space, or valvular disease. A chest X-ray revealed pulmonary edema. The laboratory data showed increased CK (1001 U/L) and troponin I serum (more than $50 \mathrm{ng} / \mathrm{mL}$ ) levels. A RMI was diagnosed, and an IABP was placed via the femoral artery to treat the cardiogenic shock. Then, an emergent coronary angi- ography was performed and it revealed no significant stenosis in the right coronary artery (Figure 5A) and left circumflex artery but rather a total occlusion in the mid LAD (Figure 5B), which coincided with the 12-lead ECG and echocardiography. We immediately moved to a primary percutaneous coronary intervention of the mid LAD with a drug-eluting stent with a TIMI grade 3 flow (Figure $5 \mathrm{C}$ ). After that, the patient became asymptomatic and hemodynamically stable during the first two days of hospitalization. On the third day, cardiac auscultation in the morning rounds revealed a newly harsh pansystolic murmur in the apex area, TTE revealed a VSR in the apex (Figure 6A), and color Doppler revealed a shunt between the left and right ventricles through the defect (Figure 6 

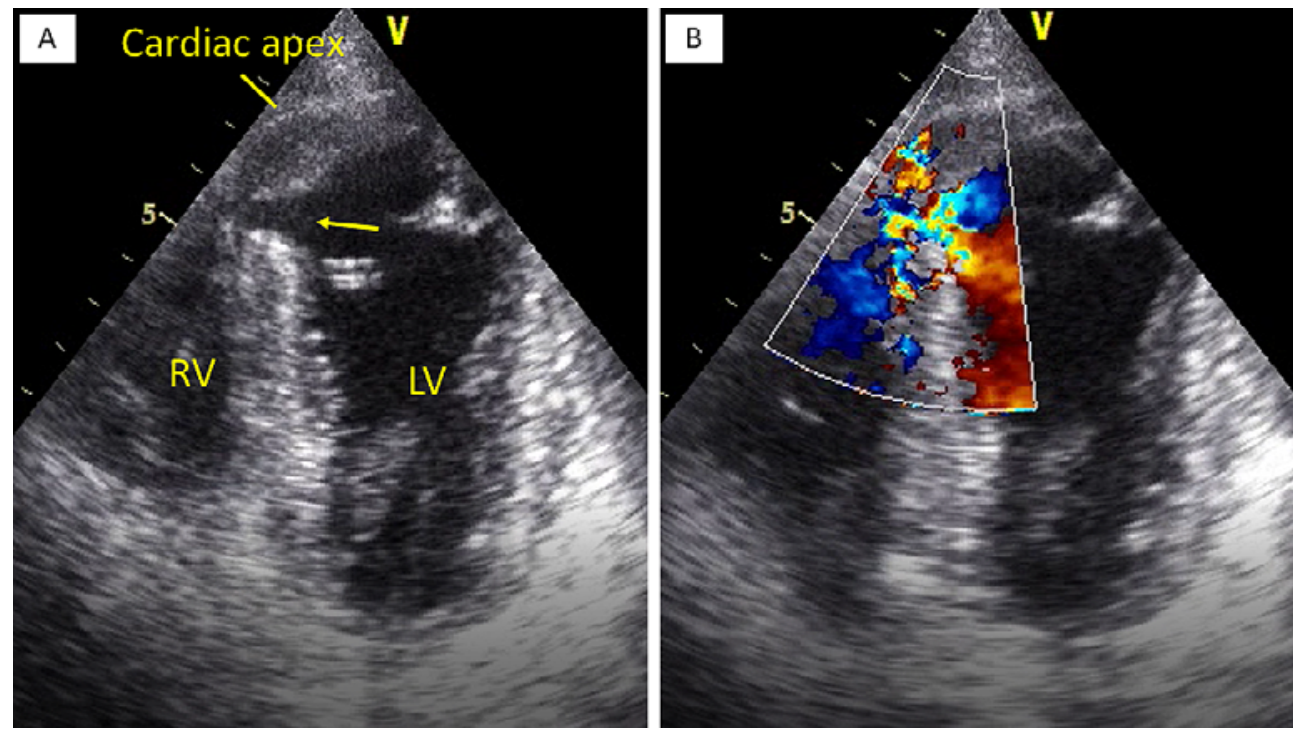

Figure 6. Transthoracic echocardiography. A: Presence of a ventricular septal defect as seen in the apical two-chamber view (arrow). B: Color Doppler showing turbulent flow (mosaic) from the left ventricle (LV) to right ventricle (RV) through the interventricular septal defect.

B). The pulmonary artery systolic pressure was $49 \mathrm{mmHg}$ on IABP and worsened to $55 \mathrm{mmHg}$ once the IABP was set to the standby mode. The $\mathrm{Qp} / \mathrm{Qs}$ ratio was 1.5 . A chest X-ray displayed an exacerbation of the congestion. We diagnosed him with acute decompensated heart failure followed by a VSR after a RMI. Based on the experience obtained in Case 1, even though the patient's surgical complication risk was very high (EuroSCORE II: $21.3 \%$, JapanSCORE: 30-day operative mortality [CABG only/ valve] $13.4 \% / 68.0 \%$, and plus major complications $52.6 \%$ / $88.3 \%$ ) (Table), our heart team suggested to the patient and his family performing surgery, and they accepted it. Because of not only an exacerbation of the congestion in spite of IABP support but also xeroderma with a history of cellulitis, which may increase the risk of puncture-site complications with the duration of IABP use, the operation was performed on day 4 . The surgical findings revealed a simple VSR. There was a 1-cm defect in the septum; the myocardium around the perforated area was vulnerable due to myocardial necrosis (Figure 7A). After debridement of those vulnerable tissues, VSR closure was successfully performed by placing a bovine pericardium patch, and the VSR was closed (Figure 7B-D). After that, the postoperative course was good without any major complications, and it was possible to withdraw the patient from the IABP on the day of surgery. Thereafter, optimal medical therapy, including a beta-blocker and mineralocorticoid receptor antagonist, and rehabilitation were performed, and the patient was eventually transferred to a rehabilitation hospital on postoperative day 19.

\section{Discussion}

VSR is a rare complication of MI that causes acute heart failure and/or cardiogenic shock. ${ }^{1)}$ The key of the medical and mechanical management of a VSR is after- load reduction to increase the effective left ventricular stroke volume by reducing left-to-right shunting and improving the stability of the cardiac tissue. ${ }^{1)}$ IABP provides a mechanical afterload reduction and augmentation of the cardiac output, which is recommended as Class I indication in the guidelines by the Japanese Circulation Society (JCS Joint Working Group [JCS 2018]) in patients with cardiogenic shock due to mechanical compliations after a MI. In Case 1, the IABP was carefully weaned, but the patient died suddenly two days after its removal.

After a rapid diagnosis and optimal medical and mechanical support, we need to discuss whether surgical intervention should be performed or not in these high risk very elderly patients. Although EuroSCORE II and JapanSCORE exist for risk assessment of cardiac surgery, the data for those aged 90 and over have not been established. For example, of over 20,000 patients in the EuroSCORE database, only 21 were aged over 90, and the oldest age in the EuroSCORE database was 95. ${ }^{2)}$ Therefore, the risk model may not be fully suitable for very elderly patients, and we need to consider the therapeutic strategy carefully and comprehensively. Suhino et al. suggested that hypoalbuminemia, frailty assessed by the Canadian Study of Health and Aging Clinical Frailty Scale (CSHA-CFS), and the body mass index could be the early prognostic factors in very elderly ( $\geq 85$ years) patients with an ST-elevation acute MI, and very elderly patients with a CSHA-CFS $<6$ do not have a poor prognosis. ${ }^{3}$ These findings implicated that even in very elderly patients, invasive interventions might be considered if they are not too frail. Nonetheless, once post-infarction VSR occurs, $90 \%$ of patients die within two months if surgical repair was not performed. ${ }^{4}$ Considering this as well as the two cases presented here, if possible, surgical intervention should be fully considered even if the patients are very old and frail. 

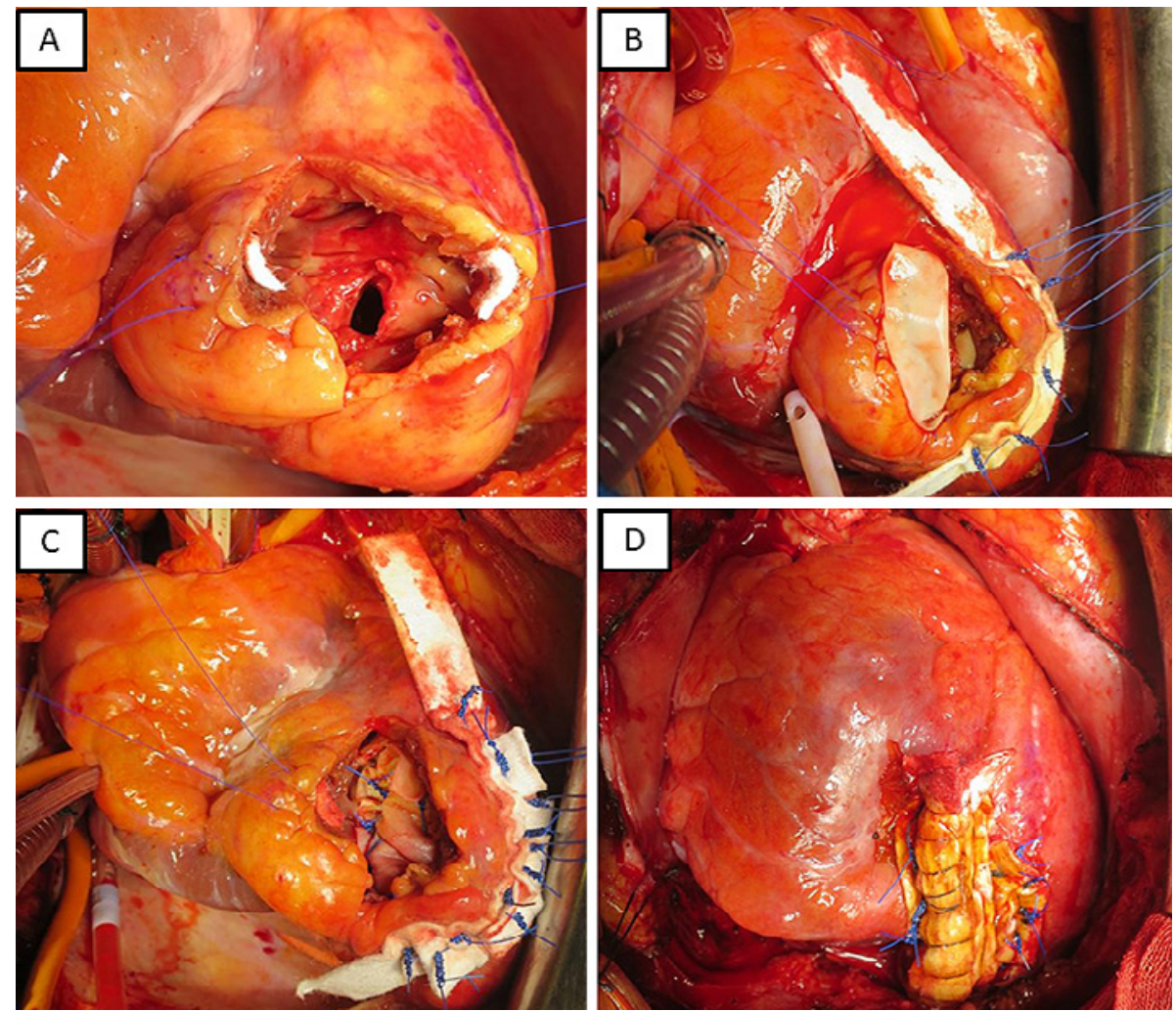

Figure 7. Inoperative findings. A: A $1-\mathrm{cm}$ septal ventricular rupture (VSR) surrounded by fragile infarcted tissue. B and C: VSR closure was performed with a bovine pericardium patch. D: Finally the right ventricle was successfully sutured.

The timing of the surgery for a VSR has yet to be discussed. The American College of Cardiology guidelines suggest that an immediate surgical repair should be performed for a VSR irrespective of the patient's hemodynamic status, ${ }^{5)}$ whereas the European Society of Cardiology guidelines suggest delayed elective surgery as an alternative in selected patients who respond to medical therapy ${ }^{6}$ because delayed surgery in patients with a VSR who respond to medical and mechanical therapy seem to have a better outcome. ${ }^{4}$ In Case 2, despite aggressive medical and mechanical treatment, the heart failure became exacerbated, and long-term IABP and an arterial line placement increased the risk of skin infections due to his xeroderma with a history of cellulitis, so a surgical repair was performed on day 4 . The surgical findings revealed a fragile myocardium around the VSR, which was successfully debrided, followed by a bovine pericardial patch and successful surgery.

In Case 1, after considering the risks of surgery, conservative treatment was selected, but the patient did not survive. On the other hand, in Case 2, whose general condition was worse than that of Case 1 , surgery was performed and his life was eventually saved; however, there were several differences between these two cases regarding the nutrition level of the patients and timing of the onset and severity of the VSR (Table).

\section{Conclusion}

Here, we describe two cases of VSR after RMI in two very elderly patients. We concluded that even if the patients with VSR are very old, when possible, surgical intervention should be fully considered.

\section{Acknowledgments}

We thank all the doctors and medical staff who were involved in the treatment of these patients. We also thank Mr. John Martin for help with the English editing.

\section{Disclosure}

Conflicts of interest: Yasuo Okumura received research funding from Boston Scientific Japan.

\section{References}

1. Jones BM, Kapadia SR, Smedira NG, et al. Ventricular septal rupture complicating acute myocardial infarction: a contemporary review. Eur Heart J 2014; 35: 2060-8.

2. Nashef SA, Roques F, Sharples LD, et al. EuroSCORE II. Eur J Cardiothorac Surg 2012; 41: 734-44.

3. Sujino Y, Tanno J, Nakano S, et al. Impact of hypoalbuminemia, frailty, and body mass index on early prognosis in older patients $(>/=85$ years $)$ with ST-elevation myocardial infarction. J Cardiol 
2015; 66: 263-8.

4. Arnaoutakis GJ, Zhao Y, George TJ, Sciortino CM, McCarthy PM, Conte JV. Surgical repair of ventricular septal defect after myocardial infarction: outcomes from the Society of Thoracic Surgeons National Database. Ann Thorac Surg 2012; 94: 43643.

5. O'Gara PT, Kushner FG, Ascheim DD, et al. 2013 ACCF/AHA guideline for the management of ST-elevation myocardial infarction: a report of the American College of Cardiology Foundation/American Heart Association Task Force on Practice
Guidelines. Circulation 2013; 127: e362-425.

6. Ibanez B, James S, Agewall S, et al. 2017 ESC Guidelines for the management of acute myocardial infarction in patients presenting with ST-segment elevation: The Task Force for the management of acute myocardial infarction in patients presenting with ST-segment elevation of the European Society of Cardiology (ESC). Eur Heart J 2018; 39: 119-77.

7. Kunimura A, Ishii H, Uetani T, et al. Impact of Geriatric Nutritional Risk Index on cardiovascular outcomes in patients with stable coronary artery disease. J Cardiol 2017; 69: 383-8. 